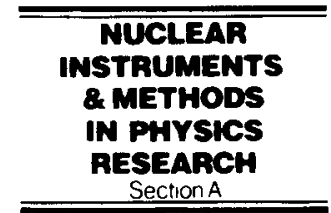

ELSEVIER

\title{
A combined video and gamma ray imaging system for robots in nuclear environments
}

\author{
R. Redus ${ }^{\text {a,* }}$, M. Squillante ${ }^{\text {a }}$, J. Gordon ${ }^{\text {a }}$, G. Knoll ${ }^{\text {b }}$, D. Wehe ${ }^{\text {b }}$ \\ ${ }^{d}$ Radiation Monttoring Devices, Inc., 44 Hunt St., Watertown, MA 02172, USA \\ ${ }^{\mathrm{b}}$ Department of Nuclear Engineering, University of Michigan, Ann Arbor, MI 48109, USA
}

\begin{abstract}
An integrated imaging sensor system is being developed to enhance operations of robots and telerobotic systems used in nuclear industry. This system combines a gamma ray image of the distribution of radioactivity with a video image of the area, allowing a rapid and intuitive determination of the source location. The gamma ray imaging system is based upon a position sensitive photomultiplier tube, a segmented scintillator, and a pinhole collimator which provides high quality images in the energy range of 0.1 to $1.5 \mathrm{MeV}$. The design and measured performance of a prototype of this system will be discussed.
\end{abstract}

\section{Introduction}

Robots are becoming increasingly important throughout the nuclear industry [1]. They are commonly used to improve operations and maintenance, lower operating costs, and reduce exposure to workers [2]. However, the capabilities of existing robots are limited by the systems used to sense their surroundings, which often include visual imaging systems and nuclear survey instruments. These survey instruments are useful for making rapid, discrete point measurements of radiation intensity but do not give directional information regarding the radiation [3]. This makes locating a source cumbersome and time-consuming and does not allow the robot or operator to easily visualize the distribution of radioactivity in the local environment. We are developing an instrument which will overcome these difficulties by combining a video camera with a portable gamma ray imaging system to produce an image of the radioactivity, superimposed on a video image of the same field of view, allowing an operator to rapidly and remotely locate radioactive sources. We have developed a prototype of such an instrument which has been used to image isotopic sources with energies between 0.1 and $1.5 \mathrm{MeV}$. We have also developed a Monte Carlo model to simulate the performance of this instrument in the presence of the radiation fields found in the nuclear industry [4]. We anticipate achieving an angular resolution for the nuclear image of $6^{\circ}$, or $1 \mathrm{~m}$ spatial resolution at $10 \mathrm{~m}$, with an

\footnotetext{
* Corresponding author.
}

efficiency of $3 \times 10^{-6}$ at $1 \mathrm{~m}$, which is suitable for many nuclear applications.

\section{Prototype design}

The prototype imager combines a gamma ray imaging system with a conventional video camera and personal computer-based data acquisition and display system. A block diagram of the prototype is shown in Fig. 1. The gamma ray imager is based upon a position sensitive photomultiplier tube (PSPMT) coupled to a scintillator and collimator. A PSPMT is a unique photomultiplier tube which combines the sensitivity and energy resolution of conventional PMTs with the ability to obtain two-dimensional position information [5]. Most importantly, because the PSPMT is only 3 in. in diameter, the gamma ray imager is sufficiently small and light to be used with many existing robots. The characteristics of PSPMTs have been discussed by many authors [6,7]. The PSPMT is a single photon imaging device, with each incident light pulse producing four analog output pulses from which the position and energy of the incident gamma ray can be computed. The energy resolution is comparable to that obtained by a conventional PMT although the gain varies significantly with position. The spatial resolution is inversely proportional to the square root of the number of optical photons per unit area incident on the photocathode.

In order to obtain positional information a collimator must be used to restrict the solid angle which may illuminate an image pixel. In the prototype instrument use a 3 


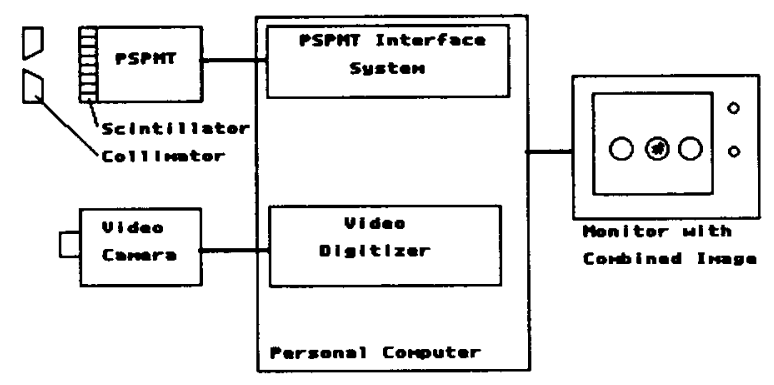

Fig. 1. Block diagram of prototype video/gamma ray imaging system.

$\mathrm{cm}$ thick lead pinhole collimator with a conical hole, with the base facing towards the scintillator (see Fig. 1). The cone allows imaging throughout the field of view with no attenuation. A pinhole collimator has several advantages over other types, including ease of fabrication, ease of operation, and angular resolution independent of distance which permits straightforward comparison with the video image. The main factor determining the efficiency and spatial resolution of the system is the angle subtended by the collimator pinhole. The distance from the front of the collimator to the PSPMT determines the angular field of view, while the diameter of the pinhole determines the angular resolution and geometric efficiency. At higher energies, the effective pinhole diameter is increased due to penetration near the edges of the pinhole.

Diverging hole collimators can also be used for imaging high energy sources when designed to provide constant angular resolution. Such a collimator should be designed with the fields of view of individual holes overlapping only at infinity $[8,9]$. In the limit where the distance between the source and collimator are large relative to collimator thickness, diverging hole and pinhole collimators, with the same hole size and the same distance between the scintillator and collimator face, have the same angular resolution and geometric efficiency. The multiple hole collimator does have some potential advantages. High energy gamma rays will penetrate the edges of the conical pinhole, causing penumbral penetration, which results in reduced resolution. This effect is larger than septal penetration in the cylindrical holes of a multiple hole collimator at the same energy. In addition, in the pinhole radiation can scatter from the aperture throughout the image plane, while scattering from the multiple hole collimator only affects a single hole. The multiple hole collimator is however much harder to fabricate, and as shown below, a pinhole collimator provides sufficient image quality for most applications of interest.

Other collimator geometries are possible. For example, a coded aperture collimator can be used with the PSPMT and would lead to increased geometric efficiency but this does not automatically improve the signal-to-noise ratio. For point sources in the absence of background radiation, coded aperture methods reduce the noise in pixels with a strong signal. However, for broadly distributed source distributions and point sources in the presence of significant background radiation, coded aperture collimators result in higher statistical noise everywhere compared with conventional imaging [10]. A coded aperture collimator is generally not advantageous in the radiation fields experienced by robots in nuclear operations.

A scintillator is used to convert the energy of the incident gamma rays into optical photons which can be detected by the PSPMT. In order to provide good stopping power for gamma rays with energy above $1 \mathrm{MeV}$ it is necessary to use a thick scintillator. The resolution of the $\mathrm{X}$-ray interaction at the PSPMT photocathode is significantly degraded with a thick conventional scintillator because of light spreading in the scintillator. For this reason, a segmented scintillator was used. The scintillator was made of BGO, $1 \mathrm{~cm}$ thick, with segments $2.5 \mathrm{~mm}$ on centers, separated by (MgO) optical reflector material. The scintillator was fabricated by ScinTech, Inc. Photons generated within a given segment are always distributed uniformly over a $6.25 \mathrm{~mm}^{2}$ area of the photocathode. For energies between 0.1 and $1 \mathrm{MeV}$ the measured PSPMT spatial resolution varies between 1 and $2.5 \mathrm{~mm}$. The segment size, which determines the maximum pixel size, is half the dimension of the pinhole, to obtain good statistics and good resolution for photon energies $>0.1 \mathrm{MeV}$.

\section{Experimental results}

In the present laboratory prototype system the PSPMT is interfaced to a personal computer using a commercially available PSPMT Interface System [11]. This system acquires digitized coordinate and energy values, implements a position dependent energy discrimination and a uniformity correction to compensate for the non-uniformity of the PSPMT [12], and places the nuclear image into a buffer in the computer. The video image is acquired using a conventional video camera and an IDEC-8 video digitizer card. The video image is displayed in gray scale on the monitor, while the nuclear image is superimposed on the video image in real-time. The nuclear image is displayed in color to make it easier to locate the source.

Fig. 2 shows images of several isotopic sources, located at $1 \mathrm{~m}$ distances, which were obtained with the prototype instrument. All three images were obtained with the same collimator and scintillator, with only the energy discrimination changed between images. Fig. $2 \mathrm{a}$ shows $80 \mu \mathrm{Ci}$ of ${ }^{60} \mathrm{Co}$, with energy emissions at 1.17 and $1.32 \mathrm{MeV}$, imaged at $1 \mathrm{~m}$ in $20 \mathrm{~min}$. Fig. $2 \mathrm{~b}$ shows $1 \mathrm{mCi}$ of ${ }^{137} \mathrm{Cs}$, with energy emission at $662 \mathrm{keV}$, imaged at $1 \mathrm{~m}$ in $30 \mathrm{~s}$. Fig. $2 \mathrm{c}$ shows $8.5 \mathrm{mCi}$ of ${ }^{57} \mathrm{Co}$, with energy emission at $122 \mathrm{keV}$, imaged at $1 \mathrm{~m}$ in $5 \mathrm{~s}$. The gamma images were processed by contrast expansion and thresholding. This clearly demonstrates the ability of the system to image a wide 

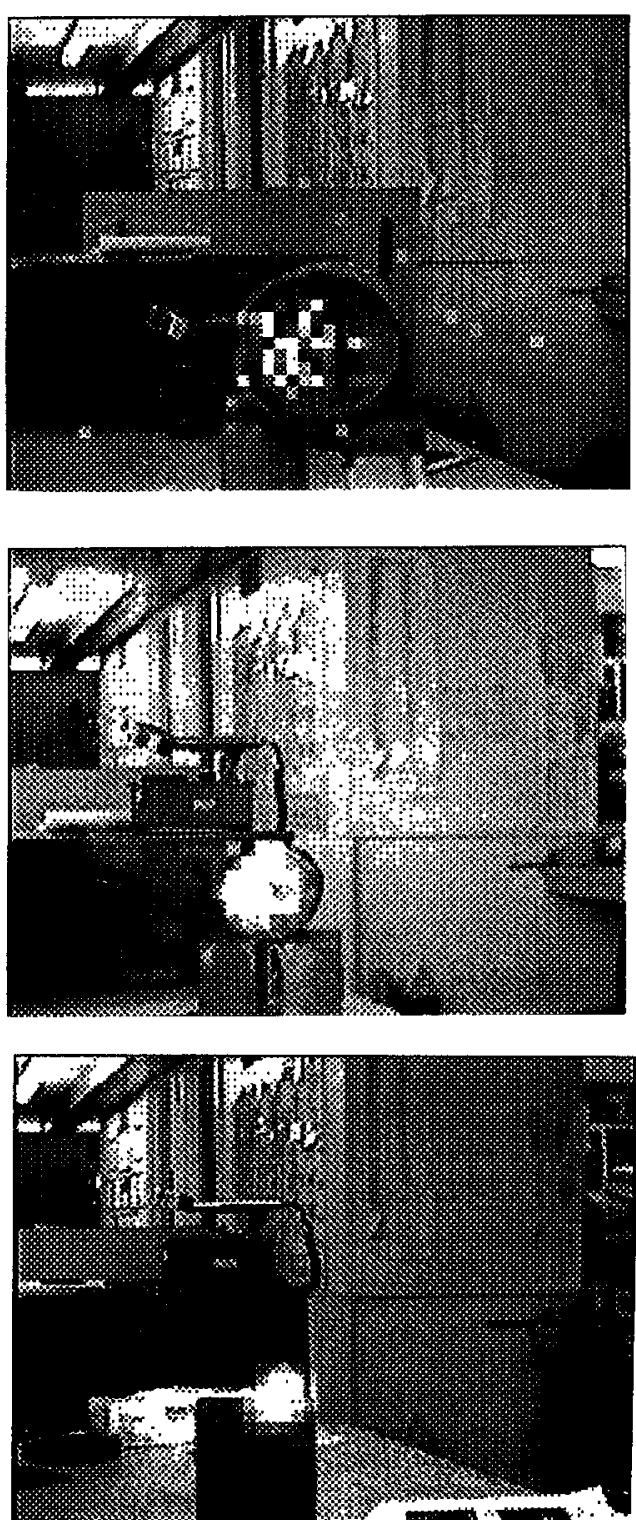

Fig. 2. Images of isotopic sources at $1 \mathrm{~m}$ distance obtained with prototype. (a) A ${ }^{60} \mathrm{Co}$ source; (b) a ${ }^{137} \mathrm{Cs}$ source, and (c) a ${ }^{57} \mathrm{Co}$ source.

range of gamma ray energies, from $122 \mathrm{keV}$ to $1.3 \mathrm{MeV}$. Fig. 3 shows a cross-section through the nuclear data for the ${ }^{57} \mathrm{Co}$ and ${ }^{137} \mathrm{Cs}$ images. At higher energies there is some additional penetration through the conical aperture in the collimator, reducing spatial resolution.

Energy discrimination is quite important in rejecting scattered radiation, in order to obtain high quality images. Because the gain of the PSPMT varies dramatically with position, software is used to carry out a separate pulse-

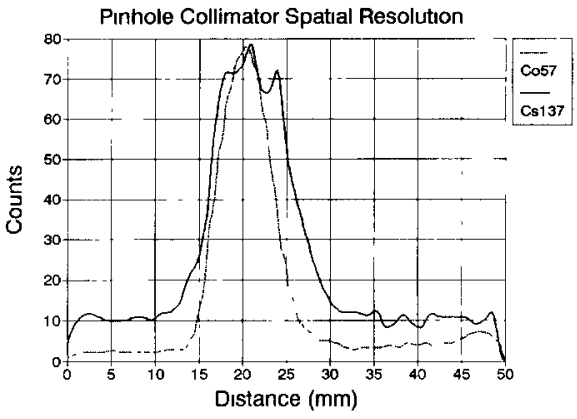

Fig. 3. Cross-section of gamma ray images obtained with prototype.

height discrimination for each spatial pixel. In this way only events within a $\pm 15 \%$ window around the photopeak are accepted in each pixel. This greatly reduces the radiation which scatters off surrounding objects and off the collimator. In addition it allows one to image a high energy source, such as the 1.17 and $1.33 \mathrm{MeV}$ lines of
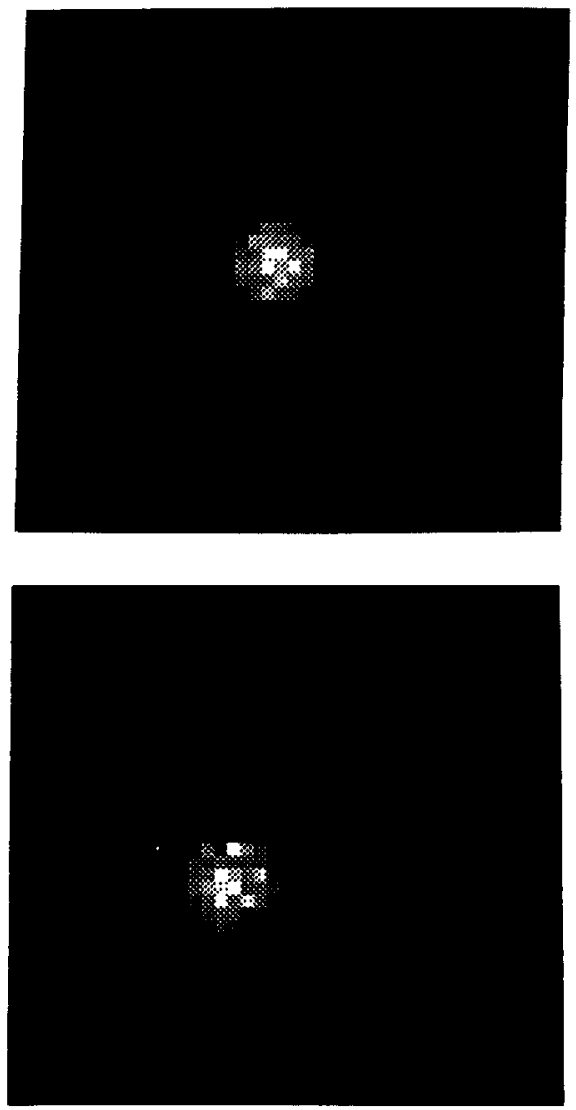

Fig. 4. Comparison of actual and simulated images of a ${ }^{137} \mathrm{Cs}$ source obtained for the same field of view. 
${ }^{60} \mathrm{Co}$, in the presence of $662 \mathrm{keV}{ }^{137} \mathrm{Cs}$. The software can easily be modified to accept multiple energy windows, simultaneously displaying gamma rays from two or more sources, or to display separate nuclear images for each source. In applications where the energy of the source is not known it would be possible to operate without energy discrimination but this would degrade image quality.

\section{Modeling results}

In order to optimize the performance of the system a Monte Carlo model of the collimator and scintillator was developed using the EGS4 computer code [13]. The source photons were generated within a cone, where the base of the cone formed a circle on the collimator. Secondary electrons were followed to $300 \mathrm{keV}$, photons to $25 \mathrm{keV}$. The model image was formed from all events which deposited, in the BGO scintillator, an energy exceeding a threshold which was set above the Compton edge. The centroid of the spatial distribution of the energy deposited in the scintillator was computed and taken as the coordinate of the event. Fig. 4 compares a simulated image of ${ }^{137} \mathrm{Cs}$ along with an actual image obtained by the prototype, for the same field of view and source geometry. The statistics are better in the simulation than in the actual image. The model clearly reproduces the spatial distribution of incident gamma rays.

The main factor determining the spatial resolution of the system is the angle subtended by the collimator pinhole, although at high energies penumbral penetration through the edges of this hole must be considered. For example, consider a collimator which is made from $4 \mathrm{~cm}$ of tungsten with a $7 \mathrm{~mm}$ pinhole, adjusted to provide a $7 \mathrm{~m}$ field of view at $10 \mathrm{~m}$ distance. At $100 \mathrm{keV}$ the angular resolution is $5.2^{\circ}$, while at $1 \mathrm{MeV}$ the angular resolution is $6.5^{\circ}$ due to penumbral penetration. The geometric efficiency is also determined by the solid angle subtended by the pinhole, as seen from the source. In our example the extrinsic efficiency is $3 \times 10^{-6}$ at $1 \mathrm{~m}$. The image acquisition time is determined by the requirement that, for the pixels containing the source, the signal to noise ratio exceeds a threshold of three. The noise is the uncertainty in the number of counts in a pixel, and since the count rate depends upon the background radiation spectrum and intensity, the image acquisition time depends upon background conditions. We estimate that our example system can image a $1 \mathrm{mCi}, 1 \mathrm{MeV}$ source at $10 \mathrm{~m}$ to $6^{\circ}$ in $25 \mathrm{~s}$ in the absence of background. In a $0.5 \mathrm{R} / \mathrm{h}$ background, with a $1 / E^{2}$ energy spectrum, we estimate that a $15 \mathrm{mCi}, 1$ $\mathrm{MeV}$ source at $10 \mathrm{~m}$ can be imaged in $25 \mathrm{~s}$.

\section{Summary and conclusions}

An integrated imaging sensor system, which combines a gamma ray image of the distribution of radioactivity with a video image of the area, is being developed. The gamma ray imaging system is based upon a position sensitive photomultiplier tube, a segmented BGO scintillator, and a pinhole collimator which provides high quality images from 0.1 to $1.5 \mathrm{MeV}$. A prototype of the system was fabricated and tested and a Monte Carlo model developed to optimize performance. We have acquired good quality images with gamma ray energies from 0.1 to $1.3 \mathrm{MeV}$. This system has demonstrated the spatial resolution and efficiency required for many applications in nuclear industry.

The results discussed here were obtained using a bench-top laboratory prototype. In the next phase of this project we will design and build a more compact system which is suitable for actual robotic use and will test it in realistic nuclear applications.

\section{References}

[1] E.B. Silverman, G.W. Boughman, F. Marian and S.K. Hamilton, Proc. 36th Conf. on Remote Systems Technology, Vol. 1 (1988) p. 13

[2] J. Bahren et al., Proc. 1984 Meeting on Robotics and Remote Handling in Hostile Environments, 1984.

[3] G.F. Knoll, Radiation Detection and Measurement (Wiley, New York, 1989).

[4] J. Ottaviani and D. Wehe, Annals Nucl. Energy 16 (1989) 91.

[5] H. Kume, S. Muramatsu and M. Iida, IEEE Trans. Nucl. Sci. NS-33 (1) (1986) 488.

[6] N.J. Yasillo, R.N. Beck and M. Cooper, IEEE Trans. Nucl. Sci. NS-37 (1) (1990) 609.

[7] J. Gordon, R. Redus, V. Nagarkar and M. Squillante, SPIE Proc. 1734 (1992) 187.

[8] S.V. Guru, J. Valentine, D. Wehe and G. Knoll, Conf. Record for the IEEE Nuclear Science Symp. '93.

[9] S.V. Guru, Z. He, J.C. Ferreria, D.K. Wehe and G.F. Knoll, these Proceedings (1994 Symposium on Radiation Measurements and Applications, Ann Arbor, MI, USA) Nucl. Instr. and Meth. A 353 (1994) 328.

[10] G.F. Knoll, W. Rogers, K. Koral, J. Stamos and N. Clinthorne, Nucl. Instr. Meth. 221 (1984) 226.

[11] R.H. Redus, V. Nagarkar, L.J. Cirignano, W. McGann and M.R. Squiliante, IEEE Trans. Nucl. Sci. NS-39 (4) (1992) 948.

[12] C.E. Ordonez, R.A. Mintzer, J.N. Aarsvold, N.J. Yasillo and K.L. Matthers, Conf. Record for the IEEE Nuclear Science Symp. '93.

[13] W.R. Nelson, H. Hirayama and D.W.O. Rogers, Stanford Linear Accelerator Center Report SLAC-265 (1985) 